\title{
Consequences of the Solar System passage through dense interstellar clouds
}

\author{
A. G. Yeghikyan ${ }^{1,2}$ and H. J. Fahr ${ }^{1}$ \\ ${ }^{1}$ Institute of Astrophysics and Extraterrestrial Research, University of Bonn, Germany \\ ${ }^{2}$ Byurakan Astrophysical Observatory, Armenia
}

Received: 12 September 2002 - Revised: 28 April 2003 - Accepted: 13 May 2003

\begin{abstract}
Several consequences of the passage of the solar system through dense interstellar molecular clouds are discussed. These clouds, dense (more than $100 \mathrm{~cm}^{-3}$ ), cold $(10-50 \mathrm{~K})$ and extended (larger than $1 \mathrm{pc}$ ), are characterized by a gas-to-dust mass ratio of about 100 , by a specific power grain size spectrum (grain radii usually cover the range 0.001-3 micron) and by an average dust-to-gas number density ratio of about $10^{-12}$. Frequently these clouds contain small-scale (10-100 AU) condensations with gas concentrations ranging up to $10^{5} \mathrm{~cm}^{-3}$. At their casual passage over the solar system they exert pressures very much enhanced with respect to today's standards. Under these conditions it will occur that the Earth is exposed directly to the interstellar flow.

It is shown first that even close to the Sun, at $1 \mathrm{AU}$, the cloud's matter is only partly ionized and should mainly interact with the solar wind by charge exchange processes. Dust particles of the cloud serve as a source of neutrals, generated by the solar UV irradiation of dust grains, causing the evaporation of icy materials. The release of neutral atoms from dust grains is then followed by strong influences on the solar wind plasma flow. The behavior of the neutral gas inflow parameters is investigated by a 2-D hydrodynamic approach to model the interaction processes. Because of a reduction of the heliospheric dimension down to $1 \mathrm{AU}$, direct influence of the cloud's matter to the terrestrial environment and atmosphere could be envisaged.
\end{abstract}

Key words. Interplanetary physics (heliopause and solar wind termination; interplanetary dust; interstellar gas)

\section{Introduction}

The interstellar medium (ISM) in our galaxy, a mixture of gas and dust is known to be highly inhomogeneous with respect to density and temperature. It tends to concentrate near

Correspondence to: A. G. Yeghikyan

(aray@astro.uni-bonn.de) the galactic plane and along the spiral arms and is composed of a dense, neutral and molecular material, embedded in an ambient warm and tenuous medium (for an overview of the composition of the ISM, see McKee, 1995). Roughly half the ISM mass is confined to discrete clouds occupying only $\sim 1-2 \%$ of the galactic disk volume. The dense component consists of cold atomic HI clouds $(T \sim 100 \mathrm{~K})$ and giant molecular clouds (GMC) $(T \sim 10-20 \mathrm{~K})$ with clumpy substructure. These clouds have irregular shapes and overall dimensions and densities ranging from $0.01-50 \mathrm{pc}$ and from $10-100 \mathrm{~cm}^{-3}$ (HI) and about of $1000 \mathrm{~cm}^{-3}$ (GMC clouds), respectively. Cores of GMC and clumps within GMC are denser (more than $10^{3}$ and $10^{5} \mathrm{~cm}^{-3}$, correspondingly), but they occupy only $\sim 0.1$ and $\sim 1 \%$ of the GMC volume, respectively (Allen, 2000).

The possibility of solar encounters with dense interstellar clouds (IC) with particle concentrations about $10-$ $1000 \mathrm{~cm}^{-3}$ and more, is of great interest in view of its possible effects upon the Earth. The question of whether dense IC would prevent the solar wind (SW) from reaching the Earth with the result of cloud material directly impacting the terrestrial atmosphere, as well as many other aspects of this complex problem were already discussed in the literature of the past (Fahr, 1968a, b; Talbot and Newman, 1977; Holzer, 1977; Fahr, 1980; Ripken and Fahr, 1981; Zank and Frisch, 1999; Scherer, 2000; Scherer et al. 2002, and references therein). Such a scenario is considered as possibly triggering global glaciations, depositions of a prebiotic material on the primordial Earth, possible ecological repercussions for the Earth due to an accretion of the cloud's matter, and of course, bio-mass extinction (Yabushita and Allen, 1997). It should also be added that in fact the correlation between periods of glaciations and long-time variations in the accretion rate of interplanetary dust particles onto the Earth is revealed (Farley and Patterson, 1995).

The mean travel time $\tau$ between encounters of the Sun with IC, a key parameter in the discussed situation, has been estimated in many works (e.g. Talbot and Newman, 1977; Scoville and Sanders, 1986; Clube and Napier, 1986), with 
the help of available observational data on the distribution of neutral and molecular hydrogen in the galaxy and refined statistical analysis. Summarizing, the results of such estimations may be represented as follows:

$\tau=\frac{\delta}{\bar{v}}$

where $\delta$ is a mean free path between encounters and $\bar{v}$ - rms velocity of the Sun relative to the IC, that is the quadratic mean of the cloud-cloud velocity dispersion and the solar peculiar velocity. It is straightforward to estimate $\delta$ roughly as

$\delta=\frac{1}{\pi D_{I C}^{2} N}$,

where $D_{I C}$ is the radius of the IC and $N$ is the number density of clouds. Adopting mean values for the parameters $D_{I C}$ and $N\left(2\right.$ and $5 \mathrm{pc}$, and $15 \cdot 10^{-5}$ and $0.14 \cdot 10^{-5} \mathrm{pc}^{-3}$ for the HI and GMC, respectively, (Talbot and Newman, 1977), as well as for the $\bar{v} \sim 20 \mathrm{~km} / \mathrm{s}$, (Scoville and Sanders, 1986), one may finally find $27 \mathrm{Myr}$ and $460 \mathrm{Myr}$ as the mean travel time between encounters of the Sun with the neutral HIand molecular $\mathrm{H}_{2}$-clouds, respectively. Scoville and Sanders (1986) have mentioned that the actual time between collisions with GMC will be $\sim 2$ times larger $(\sim 1 \mathrm{Gyr})$, while Clube and Napier (1986) have argued for shorter periods, of about 700 Myr.

Thus, one may conclude that neutral HI clouds, having a mean density of 10 to $100 \mathrm{~cm}^{-3}$, and a radius of about a few pc, are objects frequently encountered by the Sun during its galactic journey (more than 100 times since its birth $\sim 4.6$ Gyr ago), while the more dense GMC must have been encountered just a few times.

Nevertheless, Yabushita and Allen (1983), Yabushita and Allen (1989) and Yabushita and Allen (1997) have pointed out that just such a rare passage through the core of the GMC may have substantially depleted the oxygen of the Earth's atmosphere to cause the bio-mass extinction at the Cretaceous/Tertiary boundary, and only such a cataclysmic (not catastrophic) event is compatible with a well-known high concentration of iridium in a clay layer at this boundary. Finally, Wimmer-Schweingruber and Bochsler (2000) have recently interpreted an implanted gas sticking in surface layers of lunar soil grains as a record due to the encounter with a dense interstellar cloud.

To investigate the behavior of neutral matter inside a heliopause, which is a separatrix between the solar wind and the ambient interstellar medium, the interaction of the solar wind with the countermoving flow usually is treated as the problem of the penetration of neutral atoms from the local ISM (LISM) into the heliosphere (see Zank, 1999, for a recent review). To incorporate as many relevant processes of the interaction between two counterstreaming flows as possible recent models were based on numerical multifluid hydrodynamical simulations (Baranov and Malama, 1993; Pauls et al., 1995; Fahr et al., 2000). The first authors followed a semi-kinetic approach, treating the neutral component on the basis of a Monte-Carlo simulation, and constructed selfconsistenly, but time-independently, the gas dynamic model of interaction, without taking into account processes of photoionization. Pauls et al. (1995) used a time-dependent hydrodynamical model to describe the mutual influence of the two components, but the effects of photoionization and gravity were also ignored. This code was also used to investigate the global structure of the heliosphere embedded in an environment with a density of neutral hydrogen increased from the present value $n_{i} \sim 0.1 \mathrm{~cm}^{-3}$ to $10 \mathrm{~cm}^{-3}$, i.e. by a factor of 100 (Zank and Frisch, 1999).

Results of 5-fluid hydrodynamic model calculations in which for the first time all dynamically relevant particle populations were self-consistently taken into account, namely solar wind- and LISM-protons, neutral hydrogen atoms, pick-up ions due to charge exchange and photoionization processes of the atoms, galactic and anomalous cosmic ray particles (GCR and ACR), are shown in Fahr et al. (2000). As shown on the basis of this model, an increase in the LISM $\mathrm{H}$-atom density by a factor of 10 (to $1 \mathrm{~cm}^{-3}$ ) pushes the termination shock inward to a region close to Saturn's orbit (Scherer, 2000). Solar gravity was also ignored in this model, because only regions with heliocentric distances larger than $3 \mathrm{AU}$, i.e. outside the corresponding accretion radius $r_{g}$ (see below, Sect. 4), were considered.

But the influence of both gravity and photoionization on the neutral hydrogen flow into the heliosphere seems to be important for quantitative determinations of the flow parameters at regions near the Earth's orbit. Because the kinetic treatment requires very time-consuming numerical codes, we, therefore, prefer a hydrodynamic modelling here. The prevailing physical conditions in the reduced heliosphere suggest that the idealized hydrodynamic equations with the inclusion of the effects of gravity and ionization can be used here (Whang, 1996). Thus, to describe the behavior of neutrals outside of the reduced heliosphere one may use a simple gasdynamic interaction model, where the system of fluid equations both for neutral material and solar wind plasma are decoupled (see Whang, 1996), but gravity and photoionization are taken into account. Such an approach was used to study the time-indepedent flow parameters in the heliosphere (Whang, 1996).

In this paper we use the closed system of fluid equations presented by Whang (1996) to calculate a 2-D gas dynamic time-dependent simulation of neutral flow parameters when the heliosphere is drastically reduced down to a dimension of about $1 \mathrm{AU}$ or less, as a consequence of the increased density of countermoving IC, i.e. 100-1000 times greater than at present. According to the above mentioned estimates, the Sun during its life has encountered more than one hundred times such HI clouds, while only a few denser GMC (i.e. $n_{i}>10^{3} \mathrm{~cm}^{-3}$ ) might have been met.

Not included in the present model are external magnetic field influences on the heliospheric structure: even at a 10fold increased field strength its pressure cannot be comparable to the dynamic pressure of the IC with moderate density $n=100 \mathrm{~cm}^{-3}$ (Parker, 1963). Moreover, such magnetic 
fields are attributed, in general, to the more dense GMC, but not to HI clouds (Heiles et al., 1991).

It should be noted here, that in its early history, the Sun was probably more active than it is now (Guinan and Ribas, 2002, and references therein). To be more correct, the Sun, some $4.6 \mathrm{Gyr}$ ago, had an initial luminosity of $\sim 70 \%$ of the present Sun and was about $200 \mathrm{~K}$ cooler, but perhaps had a UV enhancement in the range of $\approx 150-250 \mathrm{~nm}$ by a factor of 2 during the first few $10^{8} \mathrm{yr}$ (Zahnle and Walker, 1982). The values for the earlier solar wind intensity are more uncertain. They are based on observational studies of stellar winds from young solar type stars with different ages and indicate that the winds of the young Sun could possibly be 100-1000 times more intense than at present (Lammer et al., 2002). Finally, Sackmann and Boothroyd (2003) quite recently have shown that the current accurate helioseismic data are consistent with high-precision solar evolutionary models, which predict more luminous Sun with a stronger wind than envisaged by the standard model at first 1-2 Gyr since the origin of the Sun. Such power (2-3 orders of magnitude) of the solar wind encountering HI clouds would enlarge the heliosphere beyond $10 \mathrm{AU}$ and greatly increase the ionization degree of neutral matter at $1 \mathrm{AU}$, without consequences for the Earth, which itself would be considerably powered by very strong solar output. Dense GMC probably would be able to suppress the powerful solar wind inside $1 \mathrm{AU}$ due to stronger dynamic counterpressure.

In the following we will adopt the present parameters of the solar output, which is valid for the most frequent events under consideration (encounters with IC during last 2-3 Gyr), while more specific models should be developed for the more earlier ones.

\section{How to envisage the interaction with an interstellar cloud ?}

As is evident from arguments given in the previous section neutral interstellar cloud matter, when approaching the Solar System, systematically becomes more ionized by photoionization due to continuous action of the solar EUV radiation. On the other hand, at ISM H-atom densities of the order of $n_{H}=10^{2}-10^{3} \mathrm{~cm}^{-3}$, the free passage of a $1 \mathrm{keV}$ proton (i.e. solar wind proton) is restricted by charge exchange reactions to a mean free path of less than $\lambda_{e x}=\left(n_{H} \sigma_{e x}\right)^{-1}=$ $2 \cdot\left(10^{-1}-10^{-2}\right)$ AU. At such solar distances of less than 1 AU the interstellar matter is, however, essentially photoionized. Hence, at these small distances, in a first-order view, an interaction of the solar wind protons with the ionized component of the ISM, rather than with the neutral ISM component, occurs. Adopting an H-ionization degree $\epsilon$ of the ISM at the border of the plasma-plasma interaction configuration, one can then estimate a corresponding stand-off distance $L$ of the resulting heliopause by (Fahr, 2000):

$L^{2}=r_{0} r_{s h} \sqrt{\frac{\rho_{w 0}}{4 \epsilon \rho_{H}}} \frac{V_{w 0}}{V_{H}}$.
Here, $r_{0}$ is an inner solar reference distance, where the unperturbed solar wind has a density $\rho_{w 0}$ and a velocity $V_{w 0}$, respectively. The unperturbed ISM is characterized by a density $\rho_{H}$ and a velocity $V_{H}$ relative to the solar system, $r_{s h}$ denotes the termination shock distance from the Sun. The expected termination shock of the solar wind is located at:

$r_{s h}=r_{0} \sqrt{\frac{3 K(\gamma) \rho_{w 0}}{4 C_{I S M}}} V_{w 0}$,

where $C_{I S M}$ is the Bernoulli constant of the ionized ISM given by:

$C_{I S M}=\epsilon \rho_{H}\left[\frac{1}{2} V_{H}^{2}+\frac{\gamma-1}{\gamma} \frac{P_{H}}{\rho_{H}}\right]$.

Equations (1-3) are valid in the subsonic case (the Mach number of the relative IC motion is less than 1). Similar, but more complicated expressions for the supersonic motion of the IC can be found in the cited paper (Fahr, 2000).

Now on the basis of the above heliospheric dimensions the heliopause geometry is determined by:

$R(\phi)=R_{0} f(\phi)$,

where $R_{0}=r_{s h}, \phi=\pi-\theta$, and $\theta$ is the angle between the streamline and the direction of the Sun's motion. The function $f(\phi)$ can be modeled based on existing analytical approximations (Fahr et al., 1988; Ratkiewicz, 1992; Wilkin, 1996). We have used the function given by Wilkin (1996),

$R(\phi)=R_{0} \csc \phi[3(1-\phi \cot \phi)]$

and can now start our calculations assuming that the IC when approaching the Solar System, but yet outside the heliopause, is only subject to solar photoionization processes. Only after passage over the heliopause will the neutral ISM H-atom gas then become strongly subject to charge exchange processes with the solar wind protons, which here in this paper we do not intend to consistently describe. Nevertheless, we can argue that the size and shape of the heliopause derived with the equations above, practically are not influenced by the pickup ions created from ionization of neutral atoms occurring between the Sun and the heliopause/termination shock. This is due to the fact that $\mathrm{H}$-atoms undergoing charge exchange in the innerheliospheric region with a supersonic solar wind produce H-pick-up ions which not only act upon the solar wind by momentum loading, but also by their pressure gradient. It can then be shown (see Fahr and Chashei, 2002; Fahr, 2002; Fahr and Rucinski, 2002) that the effect of H-pick-up ions, behaving nearly isothermal with the solar wind expansion, is just to cancel these two oppositely directed forces and to guarantee a nearly undecelerated solar wind flow, keeping the effective ram pressure at a constant level. In this region downstream of the termination shock the charge-exchange processes of $\mathrm{H}$-atoms with solar wind protons are anyway of nearly no dynamical and thermodynamical impact, since in this subsonic plasma region H-pick-up ions hardly can be distinguished from original solar wind protons. Thus our 


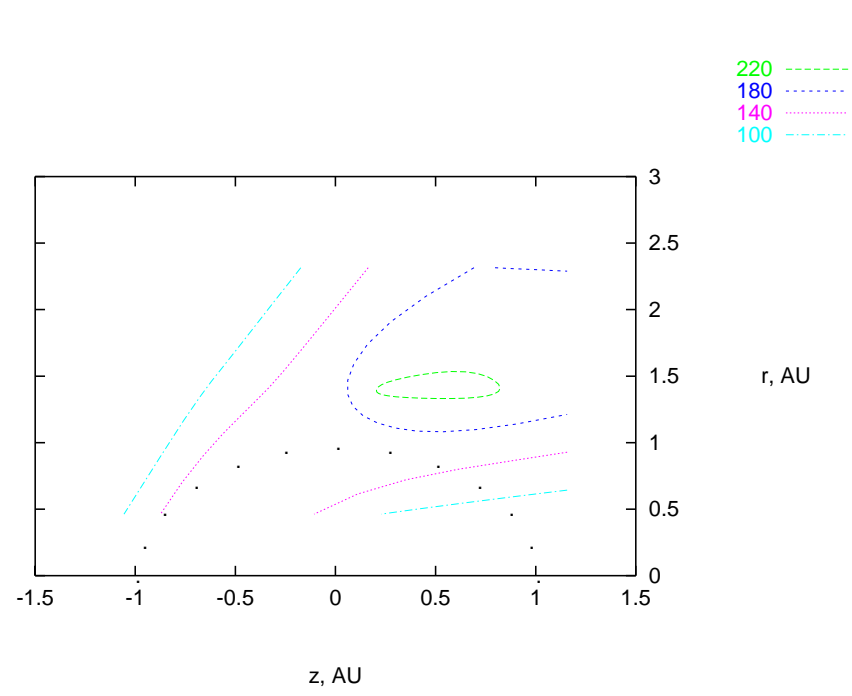

Fig. 1. Isodensity contours in the $r-z$ plane. Contours are associated to linestyles, corresponding to density values in $\mathrm{cm}^{-3}$. The Earth's orbit is shown by the dotted semi-circle. Values of the input parameters are: $n_{i}=100 \mathrm{~cm}^{-3}, V_{i}=26 \mathrm{~km} / \mathrm{s}, T_{i}=100 \mathrm{~K}$. $\beta_{0}=\beta_{\phi}, \mu=0.1$.

conclusion is that neutral $\mathrm{H}$-atoms penetrating into the heliosphere, even though not treated quantitatively here, can be expected nearly not to change the heliospheric configuration.

\section{To what extent would the circumsolar cloud be neu- tral: the static case}

The degree of ionization of the interstellar gas surrounding the Sun has been determined for modest densities in earlier works of Williams (1965) and Krasnobaev (1971) under the assumptions that there is no hydrodynamic motion (LISM at rest with respect to the Sun) and effects of the solar wind are negligible. These authors have shown similar results for the density range $n=1-500 \mathrm{~cm}^{-3}$ : gradual decrease in the ionization degree with solar distance up to a few $1000 \mathrm{AU}$, in accordance with simple estimation of the thickness of the transition region, $d \sim(a \cdot n)^{-1}$, where $a=6 \cdot 10^{-18} \mathrm{~cm}^{2}$ is the photoionization cross section of HI. In a more dense molecular environment, existing in the cores of GMC and their clumpy substructures, with densities of about $10^{5} \mathrm{~cm}^{-3}$, a sharper photodissociation region should be developed due to the fact that photodestruction of the $\mathrm{H}_{2}$ is produced by photon absorption in UV lines by, compared to $a, 2-3$ orders of magnitude larger cross sections.

Yabushita and Allen (1983) have argued that if the Sun should ever have passed through such dense core of GMC $\left(n_{H}=10^{5}\right)$, then as much oxygen as is now present in the terrestrial atmosphere could have been removed, due to water formation.

\section{Model of the neutral gas flow}

We approximate the situation as follows: let the Sun be in the origin of a system of cylindrical coordinates $(r, \zeta, z)$, and let the axis of symmetry $z$ be antiparallel to the motion of the Sun, that is the countermoving cloud matter flows from left to the right (Fig. 1), i.e. into the positive $z$-direction. The Sun affects the circumsolar flow by means of gravitation, solar wind plasma flow and solar radiation.

As is mentioned in Sect. 2, except for the interaction interface (within the range of a few free paths of the solar wind protons with respect to IC $\mathrm{H}$-atoms, say $1 \mathrm{AU}$ at $100 \mathrm{~cm}^{-3}$, and less for larger densities of neutrals), the steady-state neutral flow is completely shielded from the direct solar wind influence.

Effects of the hydrodynamical motion of the inflow upon the ionization state of the gas will be examined below. It should be underlined here that we have focused our consideration to completely neutral inflow matter and to IC densities of, and greater than, $100 \mathrm{~cm}^{-3}$. This is enough to justify the hydrodynamic approximation as close to the Sun as $1 \mathrm{AU}$, because at these densities the mean free path of the particles is less than $1 \mathrm{AU}$ due to collisions between them with cross sections of more than $10^{-15} \mathrm{~cm}^{2}$.

To describe the behavior of the neutral flow, as a first step, one can choose simply a single-fluid model, which is a set of conservation laws for mass-momentum-energy-flows with the right sides containing the corresponding source terms due to gravitation and ionization (e.g. Whang, 1996):

$$
\begin{aligned}
& \frac{\partial \rho}{\partial t}+\nabla(\rho \mathbf{V})=-\beta \rho \\
& \frac{\partial(\rho \mathbf{V})}{\partial t}+\nabla \Pi=\rho(1-\mu) \nabla\left(\frac{G M}{R}\right)-\beta \rho \mathbf{V} \\
& \frac{\partial E}{\partial t}+\nabla[\mathbf{V}(E+P)]=\rho(1-\mu) \mathbf{V} \nabla\left(\frac{G M}{R}\right)-\beta E .
\end{aligned}
$$

Here, $\rho, \mathbf{V}$ and $P$ are density, velocity and scalar pressure of neutral hydrogen, respectively, $\Pi_{i k}=\rho V_{i} V_{k}+P \delta_{i k}$, and $E=\frac{1}{2} \rho V^{2}+\frac{P}{\gamma-1}$ are the HD-stress tensor and the total energy per unit volume, respectively, $R=\sqrt{\left(r^{2}+z^{2}\right)}$ is the heliospheric distance in a cylindrical coordinate system $(r, \zeta, z)$ with symmetry axis $\mathbf{z}, \gamma=5 / 3$ is the specific heat ratio for monatomic hydrogen gas, $\mu$ is a factor denoting the ratio of the repulsion force by solar $L_{\alpha}$ radiation to the gravitational attraction force, and $M$ and $G$ are the solar mass and the gravitational constant, respectively. The total ionization rate of neutral hydrogen atom by charge exchange ionization and by photoionization is approximately defined by (Whang, 1996):

$\beta=\beta_{0}\left(\frac{r_{E}}{R}\right)^{2}$,

with $\beta_{0}=\beta_{c e}=7.68 \cdot 10^{-7} \mathrm{~s}^{-1}$. The photoionization rate $\beta_{\phi}$ itself is equal to $8 \cdot 10^{-8} \mathrm{~s}^{-1}$ at the same reference point $r_{E}$. In the following we will use $\beta_{0}=\beta_{\phi}$ according to the above condition that neutral flow material in the region described in this paper is completely shielded from solar wind protons. 

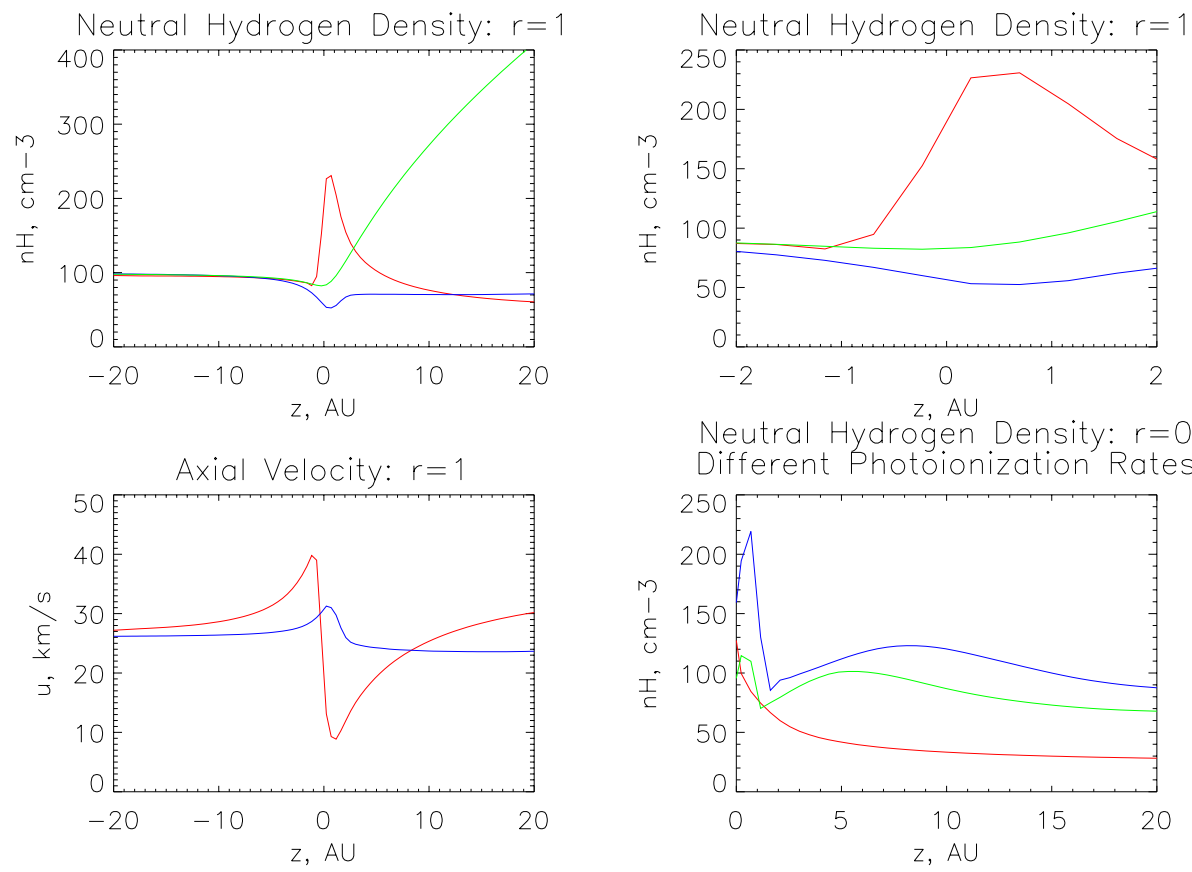

Fig. 2. Top: number density of the IC H-atom flow versus axial distanse $z$, at a radial coordinate $r=1 \mathrm{AU} . n_{i}=100 \mathrm{~cm}{ }^{-3}, V_{i}=26 \mathrm{~km} / \mathrm{s}$, $T_{i}=100 \mathrm{~K} . \beta_{0}=\beta_{\phi}, \mu=0.1$, the spatial resolution along the $z$-axis is $0.5 \mathrm{AU}$. The role of gravity is illustrated by the presence $(G \neq 0)$ and absence $(G=0)$ of the gravitational field of the Sun, shown by red and blue curves, respectively. The density distribution in the cold kinetic model (see text) with the same input parameters is shown by the green curve. Bottom, left: axial component of velocity versus $Z$ for the same parameters as in the top. Bottom, right: density distribution in downstream region, calculated for different photoionization rates is shown by red $\left(\beta_{\phi}=8 \cdot 10^{-8} \mathrm{~s}^{-1}\right)$, green $\left(5 \cdot 10^{-8}\right)$ and blue $\left(3 \cdot 10^{-8}\right)$ curves, respectively.

The flow of neutral hydrogen in the heliosphere is assumed to be symmetrical about the $z$-axis, so the results shown here can be rotated with respect to the $z$-axis.

For an axis-symmetrical case, when all derivatives in $\zeta$ vanish, using dimensionless variables

$\tilde{\rho}=\rho / \rho_{i}, \tilde{u}=u / V_{i}, \tilde{v}=v / V_{i}, \tilde{P}=P / \rho_{i} V_{i}^{2}$,

$\tilde{E}=E / E_{i}, \tilde{t}=t V_{i} / \lambda, \lambda=\beta_{0} r_{E}^{2} / V_{i}, \tilde{z}=z / \lambda, \tilde{r}=r / \lambda$,

where $u$ and $v$ are the corresponding components of velocity $\mathbf{V}$, this system has a standard vector form of a hyperbolic set of partial differential equations (LeVeque et al., 1998) with relevant source terms on the right sides given in the form:

$\mathbf{q}_{t}+\mathbf{A}(\mathbf{q})_{z}+\mathbf{B}(\mathbf{q})_{r}=\Psi(r, z, t, \mathbf{q})$.

When, omitting the tilde on top of the variables for the rest of the paper, we obtain

$$
\begin{aligned}
& \mathbf{q}=\left[\begin{array}{c}
\rho \\
\rho u \\
\rho v \\
E
\end{array}\right]=\left[\begin{array}{l}
q_{1} \\
q_{2} \\
q_{3} \\
q_{4}
\end{array}\right], \\
& \mathbf{A}(\mathbf{q})=\left[\begin{array}{c}
q_{2} \\
q_{2}^{2} / q_{1}+P \\
q_{2} q_{3} / q_{1} \\
q_{2}\left(q_{4}+P\right) / q_{1}
\end{array}\right], \mathbf{B}(\mathbf{q})=\left[\begin{array}{c}
q_{3} \\
q_{2} q_{3} / q_{1} \\
q_{3}^{2} / q_{1}+P \\
q_{3}\left(q_{4}+P\right) / q_{1}
\end{array}\right],
\end{aligned}
$$

$$
\Psi=-\left[\begin{array}{c}
q_{1} / R^{2}+q_{3} / r \\
q_{1} z \Gamma / R^{3}+q_{2} / R^{2}+q_{2} q_{3} / q_{1} r \\
q_{1} r \Gamma / R^{3}+q_{3} / R^{2}+q_{3}^{2} / q_{1} r \\
q_{2} z \Gamma / R^{3}+q_{3} r \Gamma / R^{3}+q_{4} / R^{2}+q_{3}\left(q_{4}+P\right) / q_{1} r
\end{array}\right],
$$

where

$$
\Gamma=\frac{1-\mu}{\beta} \frac{G M}{R^{2} V_{i}},
$$

is constant due to our choice of $\beta$.

The initial data describe an inner cavity with a shape given by Eq. (7) and density $\rho_{w}$, and a counterstreaming unidirectional homogeneous inflow of gas.

With the adopted values $\rho_{E} / m_{p}=5 \mathrm{~cm}^{-3}, \rho_{i} / m_{p}=100$ and $/$ or $1000 \mathrm{~cm}^{-3}$ ( $m_{p}$ is the proton mass), $V_{w}=400 \mathrm{~km} / \mathrm{s}$ and $V_{i}=26 \mathrm{~km} / \mathrm{s}$, we have solved the above system by means of the well-known LeVeque's code CLAWPACK (LeVeque et al., 1998), based on Godunov's method with high-order correction terms, on a uniform grid $160 \times 60$. Here we present results for the most important cases with countermoving IC densities $n_{i} \sim 100 \mathrm{~cm}^{-3}$ and temperatures $T_{i} \simeq 100 \mathrm{~K}$, as shown in Figs. 1-3. Our choice of $T_{i}$ is based on the fact that a dynamical timescale $\tau_{d} \sim D / V_{H} \sim 20 \mathrm{yr}$ is much less than a heating timescale $\tau_{h}$, which can be defined as $1 / \beta \sim 1 / \beta_{\phi} /(D)^{2} \sim 4000 \mathrm{yr}$, where $D \sim 100 \mathrm{AU}$ is the characteristic dimension.

Length units are in units of the ionizational length scale $\lambda$, which is equal to $0.46 \mathrm{AU}$ for the adopted parameters, and 

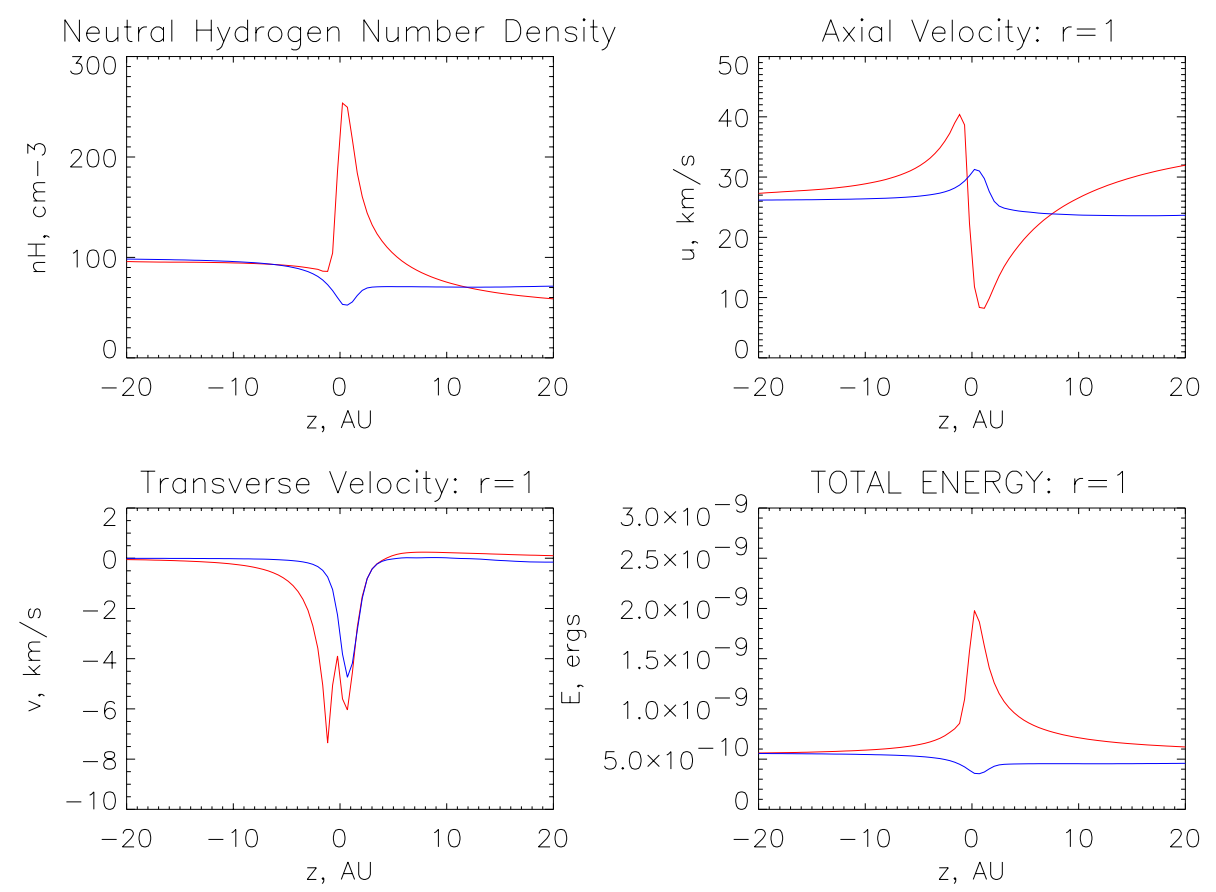

Fig. 3. Number density, axial and transverse components of velocity, and total energy of the IC H-atoms flow versus axial distance $z$, at a radial coordinate $r=1 \mathrm{AU}$. Input parameters are the same as in Fig. 2, but $\mu=0$ and 1, shown by red and blue curves, respectively.

for the calculational domain used this corresponds to a spatial resolution of $0.5 \mathrm{AU}$ along the $z$-axis. The models have been integrated forward in time until a steady configuration is reached (usually corresponding to a few hundred years).

In Fig. 1, the isodensity contours as functions of coordinates $r$ and $z$ are shown, while in Fig. 2 the distribution of density and axial component of velocity versus axial distance $z$ is represented at the corresponding radial coordinate $r=1 \mathrm{AU}$.

In Fig. 2, we compare the calculated distribution of density for different cases (gravitating, non-gravitating and lowered photoionization rates) with that of a cold (collisionless) kinetic model (e.g. Holzer, 1977, his Eq. 21) for the same input parameters and $\beta=\beta_{\phi}=8 \cdot 10^{-8} \mathrm{~s}^{-1}$. The density increase close to the Sun is evidently caused by gravity (cf. the cases $G \neq 0$ and $G=0$ in Fig. 2), whereas the strong density enhancement in the downstream region shown by the "cold" model is typical for all collisionless models, with the singular behavior of density in the tail close to the axis. It should be noted here that the enhancement of the density in the downstream flow is revealed in all 2-D hydrodynamic calculations of axisymmetric accretion flow without (Hunt, 1971; Shima et al., 1985) and with counterpressure due to the stellar wind (Shima et al., 1986). As their results show, increasing the distance from the central body and the Mach number of the flow causes the density enhancement downstream to decrease and to approach only a few units at an accretion radius $r_{g}=2 G M / V_{i}^{2}$ (Hunt, 1971). There is no point of infinite density on the axis (Shima et al., 1985). At the same time these authors have not taken into account any processes like photoionization, which is partly capable of suppressing the contrast of the tail with respect to the surrounding region. It is clearly seen in Fig. 2 (right bottom), where the density distribution for the standard case $\left(\beta_{\phi}=8 \cdot 10^{-8} \mathrm{~s}^{-1}\right)$ is compared with that calculated for lower rates $\beta_{\phi}=5 \cdot 10^{-8}$ and $3 \cdot 10^{-8} \mathrm{~s}^{-1}$. The lower the photoionization rate is, the larger is the density enhancement downstream, in accordance with the authors cited above.

The optical depth in the $L_{\alpha}$ hydrogen line, even at the $1 \mathrm{AU}$, would always be greater than 1 for $n_{i} \simeq 100 \mathrm{~cm}^{-3}$. At the same time an influence of diffuse scattered $L_{\alpha}$ photons would be negligible. The point is that only the first scattering of the solar $L_{\alpha}$ photon contributes to the radiation pressure, because multiply scattered photons become more or less isotropic and do not contribute significantly to the momentum transfer anymore, i.e. their net effect would be zero. The solar $L_{\alpha}$ profile has about a $2 \AA$ width, whereas the IC $\mathrm{H}$ atom in the front of the flow, with a radial velocity component of $40 \mathrm{~km} / \mathrm{s}$ would scatter photons, shifted only $0.2 \AA$ from the center of the line (Holzer, 1977). It evidently means that the deeper layers of the flow will not receive direct solar $L_{\alpha}$ radiation due to the absence of any considerable velocity gradient. Thus $\mu=0.1$ seems to be a fairly correct approximation. Nevertheless, to qualitatively reveal the differences between the optically thick and optically thin cases (in other words, between isotropic and non-isotropic diffuse radiation fields), the results for values $\mu$ equal to 0 and 1 , respectively, are shown in Fig. 3 (which, of course, also corresponds to the cases with and without gravity, shown in Fig. 2).

For the present inflow density of about $0.1 \mathrm{~cm}^{-3}$ our results coincide with that of the single-fluid model of Whang (1996) (with $\beta=\beta_{c e}$ ), but we can't compare them at $1 \mathrm{AU}$ 
with Zank and Frisch (1999), because these authors have presented results for an IC density of about $10 \mathrm{~cm}^{-3}$, when our approach is not valid at $1 \mathrm{AU}$. In addition, Zank and Frisch (1999) have not taken into account gravity, which is important for the simple reason that only when $R \geq r_{g}$, the flow is unaffected by gravitation (Talbot and Newman, 1977). For $V_{i}=26 \mathrm{~km} / \mathrm{s}$ one finds $r_{g}=2.6 \mathrm{AU}$. It means that the case of Bondi's flow with $\rho \simeq \rho_{i}\left(r_{g} / R\right)^{3 / 2}$ and $V^{2} \simeq 2 G M / R$ is applicable inside the distance $r_{g}$ (see Fig. 2).

We can now estimate the amount of neutral gas accreted by the Earth (an interaction between the charged particles and the magnetosphere is more complicated, and we simply assume here that all of them will be deflected). By order of magnitude accuracy one may write for the amount of accreted matter (Butler et al., 1978):

$d M / d t \simeq \pi R_{E}^{2} \cdot V_{r e l} \cdot n \cdot m_{p}(g / s)$,

where $R_{E}$ is the Earth's radius, which practically coincides with the Earth's accretion radius at relative velocity $V_{r e l}$ of the $\mathrm{H}$-atom with respect to the Earth of about $40 \mathrm{~km} / \mathrm{s}$. It is clear that there is an annual variation in $V_{r e l}$ between 10 to $70 \mathrm{~km} / \mathrm{s}$ due to the orbital velocity of the Earth, equal to about $30 \mathrm{~km} / \mathrm{s}$. An average value of $40 \mathrm{~km} / \mathrm{s}$, therefore, would be quite reasonable. Due to many uncertainties involved in the downstream region, where the direct influence of the solar wind protons is essential, we have used an upstream density value at $1 \mathrm{AU}$. For example, for $n_{i} \simeq 100 \mathrm{~cm}^{-3}$, using calculated values of $n$ (about $100 \mathrm{~cm}^{-3}$, see Fig. 2), one finds that during the pass-over time of about 1 million years the Earth may accrete about $2 \cdot 10^{16} \mathrm{~g}$ per one passage. The calculated value is by two orders of magnitude less than that estimated in Butler et al. (1978), because lower relative velocities of the encounter (about $2 \mathrm{~km} / \mathrm{s}$ with corresponding $r_{g} \sim 400 \mathrm{AU}$ ) were considered.

It is a quite accurate result up to a factor of 2 in the case of a perpendicular inflow to the ecliptic plane, and is a lower limit in other cases, because we have not taken into account here the density enhancement inside the $r_{g}$. We can qualitatively argue that such behavior would be the case even at the downstream side of the density maximum, because nowhere in the heliosphere does a sink mechanism for neutral atoms other than photoionization exist (electron impact ionization at $1 \mathrm{AU}$ would be negligible). Newly-created fast neutrals (due to charge exchange with solar wind protons) may also be accreted by the Earth. The quantitative investigation of such a scenario will be addressed in a forthcoming paper.

It should be pointed out that the results of our hydrodynamic model close to the downwind axis may be affected by some numerical problems related to the mesh size. Moreover, for IC modest densities of $\sim 100 \mathrm{~cm}^{-3}$, hydrodynamic treatment, as is mentioned above, is not fully valid for the distances less than $1 \mathrm{AU}$. On the other hand, for the same distances, the differences between the hydrodynamic and cold kinetic models (Fig. 2, top, right) are of the order of a factor of 2.5. Since uncertainties related to the uncertainty of the $L_{\alpha}$ radiation transport are also of about a factor of 5 or less (Fig. 3, top, left), the approximation that is obtained seems sufficient to draw the qualitative conclusions that are presented.

\section{Effects of neutral molecules generated by irradiated dusty grains}

In Sect. 4, we have assumed that only sinks are present for the neutral $\mathrm{H}$-atom flow without any sources. On the other hand, by direct analogy with comets one can't exclude gas-loading of the flow by neutral gas, generated via gas-desorption from dust grains, present in the cloud's matter and dynamically incorporated into the IC gas. Since these particles are likely to be very fast rotators (Spitzer, 1978), one can assume that the distribution of desorbed molecules is isotropic with respect to sublimated dust grains. It means that gas-loaded flow should be predominantly controlled by the source term, i.e. the conservation of mass (Eq. 8), and consequently, as a first approximation, the corresponding terms in Eqs. (9) and (10) can be neglected, as first pointed out by Biermann et al. (1967) in the case of the interaction between the solar wind and comets. Thus, only Eq. (8) needs to be rewritten as

$\frac{\partial \rho}{\partial t}+\nabla(\rho \mathbf{V})=-\beta \rho+Z_{t} m_{n}$

where $Z_{t}$ is a total gas production rate in units $\mathrm{mol} \cdot \mathrm{s}^{-1} \cdot \mathrm{cm}^{-3}$, and $m_{n}$ is a mass of newly-created molecules. Taking $\beta_{0}=\beta_{\phi}$ and assuming a timeindependent injection rate inside some $R \leq R_{c}$, we then obtain that the flow may be affected, if $Z_{t} \sim \beta_{\phi} \cdot n_{i}$, that is when the rate of gas-desorption is comparable to the rate of photoionization. The question of whether a population of dust grains in the matter of dense clouds exists, which may cause such a mass-injection rate, is the subject of the following discussion.

Dust grains when entering into the inner solar system will, at first, evaporate their volatile mantles, which according to generally assumed "core-mantle" models (e.g. Williams, 1993, see below), depending on environment conditions, predominantly consist of icy water (about $80-90 \% \mathrm{H}_{2} \mathrm{O}$, and $10-20 \% \mathrm{CO}, \mathrm{CH}_{4}, \mathrm{NH}_{3}$, etc., by the number of molecules). Due to consecutive photodissociation of water and some other species, under the influence of the Sun's irradiation, new hydrogen atoms should be inevitably created (see, e.g. Fahr et al., 1981). From now on, we will use a vaporization theory describing a sublimation from solid to gas, developed to calculate the evaporation rate of cometary nuclear ices, as the comet approaches the Sun (Delsemme, 1982). The total production rate of gas per 1 particle $Z_{0}=Z_{E} \cdot 4 \pi \mathrm{s}^{2}$, in units $\mathrm{mol} \cdot \mathrm{s}^{-1}$, depends strongly on the dust grain surface temperature $T_{d}$ and latent heat per molecule for the vaporization, rotation rate, albedo, chemical composition, etc. Here, $Z_{E}$ is the gas production rate per unit area, at $1 \mathrm{AU}$.

Results of theoretical calculations of $Z_{E}$, presented in the above cited paper (Delsemme, 1982) are given for different ices at $1 \mathrm{AU}$, per unit area of the perfectly absorbing, nonrotating nucleus. For the mentioned dominant components $\mathrm{H}_{2} \mathrm{O}, \mathrm{CO}$ and $\mathrm{CH}_{4}$ the values of gas production 
Table 1. Production rates for different grain mantle radii

\begin{tabular}{ccccc}
\hline $\begin{array}{c}\mathrm{s} \\
(\mu \mathrm{m})\end{array}$ & $\begin{array}{c}Z_{0} \\
(\mathrm{~mol} / \mathrm{s})\end{array}$ & $d_{g}$ & $\begin{array}{c}Z_{t} \\
\left(\mathrm{~mol} / \mathrm{cm}^{3} / \mathrm{s}\right)\end{array}$ & $h$ \\
\hline 0.5 & $3.1 \cdot 10^{4}$ & $3.7 \cdot 10^{-14}$ & $2.0 \cdot 10^{-6}$ & 3.5 \\
3.0 & $1.1 \cdot 10^{6}$ & $8.3 \cdot 10^{-16}$ & $1.6 \cdot 10^{-6}$ & 2.5 \\
30. & $1.1 \cdot 10^{8}$ & $5.0 \cdot 10^{-21}$ & $9.9 \cdot 10^{-10}$ & 3.5 \\
\hline \multicolumn{5}{c}{} \\
\hline$n_{i}$ & $R_{\max }$ & $\beta_{\phi} \cdot n_{i}$ & $Z_{E}$ \\
$100 \mathrm{~cm}^{-3}$ & $100 \mathrm{AU}$ & $8 \cdot 10^{-10} \mathrm{~cm}^{-3} \cdot \mathrm{s}^{-1}$ & $10^{16} \mathrm{~mol} / \mathrm{cm}^{2} / \mathrm{s}$ \\
\hline
\end{tabular}

rate $Z_{E}$ for rotating particles (that is with a factor of $1 / 4$ since the total area of the sphere is 4 times its cross section) are $0.42,3.2$ and 2.6, respectively, in adequate units $10^{18} \mathrm{~mol} \cdot \mathrm{cm}^{-2} \cdot \mathrm{s}^{-1}$. Corresponding steady-state effective temperatures of the grains, as well as heliocentric distances $R_{c}$ beyond which the vaporization rate becomes negligible, are equal to $195 \mathrm{~K}, 50 \mathrm{~K}, 39 \mathrm{~K}$, and $2.5 \mathrm{AU}, 38 \mathrm{AU}$ and 62.5 AU, respectively. Other components have corresponding values within the same range. In fact, observations of real comets show at $1 \mathrm{AU}$ the values $Z_{E} \simeq 1$. $10^{16} \mathrm{~mol} \cdot \mathrm{cm}^{-2} \cdot \mathrm{s}^{-1}$ apply both for production rates of water (comet P/Encke, albedo 0.7) and atomic hydrogen (comet Bennett 1970 II, albedo 0.7) (Delsemme, 1982). Optical properties of interstellar dust particles cause an albedo within the narrow range of 0.5-0.7 (Mathis, 1996). Strictly speaking, the thermal behavior of dust must be determined selfconsistently with that of gas and dust dynamics. In principle, $T_{d}$ may be obtained through a detailed solution of the equation of conservation of energy, including heating and cooling mechanisms. Due to the many uncertainties involved, to qualitatively describe the mass-loading by newly generated neutral molecules, appearing as the result of vaporization of inflowing dust grains, we have adopted a simple heliocentric distance-dependence according to

$Z=Z_{E} \cdot\left(\frac{r_{E}}{R}\right)^{2}$,

regardless of the type of newly-created neutral molecules is concerned. All the species evaporate with adopted gas production rate $Z_{E} \sim 10^{16} \mathrm{~mol} / \mathrm{cm}^{2} / \mathrm{s}$ at $1 \mathrm{AU}$. Mass-loading of the flow only takes place within a limited region $R_{\min } \leq$ $R \leq R_{\max }$. To calculate the total gas production rate $Z_{t}=$ $Z_{0} \cdot d_{g} \cdot n_{i}$ (in units $\mathrm{mol} \cdot \mathrm{s}^{-1} \cdot \mathrm{cm}^{-3}$ ) it is necessary to know the dust-to-gas density ratio $d_{g}=n_{d} / n_{i}$, depending on the grain size distribution law and grain radii $s$, which is a nontrivial problem. For order of magnitude estimations we have used the model of core-mantle grains with a power law size distribution

$n_{d}(s) \propto s^{-h}$,

the same form as is widely used to model the extinction curve in the general ISM (Mathis et al., 1977; Kim et al., 1994; Mathis, 1996; Bhatt, 1986; Rowan-Robinson, 1992;
Maiolino et al., 2001; Maiolino and Natta, 2002). Strictly speaking, large dust grains with volatile icy mantles, grown under the conditions of the dense core of the GMC, are absent in the usual diffuse clouds, but they may be present in a dense small-scale (5-10 AU) structure, which probably is a general property of the ISM (Frisch, 1996; Sciama, 2000, and references therein). Nevertheless, to be on the conservative side, we place a limit only on the GMC cases.

We can now estimate the gas production rate. Three populations of dust particles were analysed with different grain mantle sizes, namely, $0.5,3$ and $30 \mu \mathrm{m}$, respectively. If the total dust-to-gas ratio is known from the extinction properties of the molecular ices, one may then calculate the grain surface area per hydrogen particle averaged over the mentioned power law size distribution. For example, for particles with $s \leq 0.7 \mu \mathrm{m}$ (e.g. Rawlings et al., 1992; Smith et al., 1988), $\overline{d_{g} \cdot s^{2}} \simeq 2.6 \cdot 10^{-22}$, where $s$ is in $\mathrm{cm}$ and the slope $h=3.5$ is used to calculate the $d_{g}$. For $3 \mu \mathrm{m}$-sized particles we have extrapolated this value by means of the slope $h=2.5$ of flattened power law distribution (Maiolino et al., 2001; Maiolino and Natta, 2002). Data for the largest size population were taken from Rowan-Robinson (1992) and are consistent with $h=3.5$. Results are summarized in Table 1 , where gas production rates $Z_{t}$ are calculated for a ratio $Z / Z_{E}=10^{-4}$, which corresponds to the distance $R=R_{\max }=100 \mathrm{AU}$. It is clearly seen that (at the same distance) the rate of gas-desorption $Z_{t}$ predominates the rate of photoionization $\beta_{\phi} \cdot n_{i} \simeq 8 \cdot 10^{-10} \mathrm{~cm}^{-3} \cdot \mathrm{s}^{-1}$.

Thus, all three possible kinds of entering dust populations may affect the flow, especially the density behavior inside the solar system, which is illustrated in Fig. 4, where modest values of the ratio $\omega=Z_{t} \cdot m_{n} / \beta_{0} / \rho_{i}=1$ and 10 at $1 \mathrm{AU}$ were used.

At the same time such high gas production rates imply fast and complete sublimation of micron-sized particles long before they may reach the distance of $1 \mathrm{AU}$, because all of them only contain a limited number of molecule species, frozen in the mantle. In that case always $R_{\min }>1 \mathrm{AU}$ is valid, so the final effect would depend on the grain size distribution law.

On the other hand, the value $\omega>10^{3}$ is characteristic everywhere for the first two kinds of particles, or even for all of them, when one recalculates the data of Rowan-Robinson 

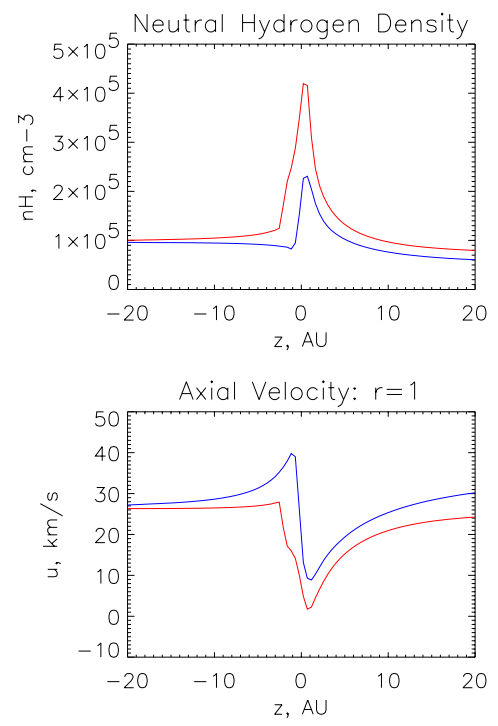
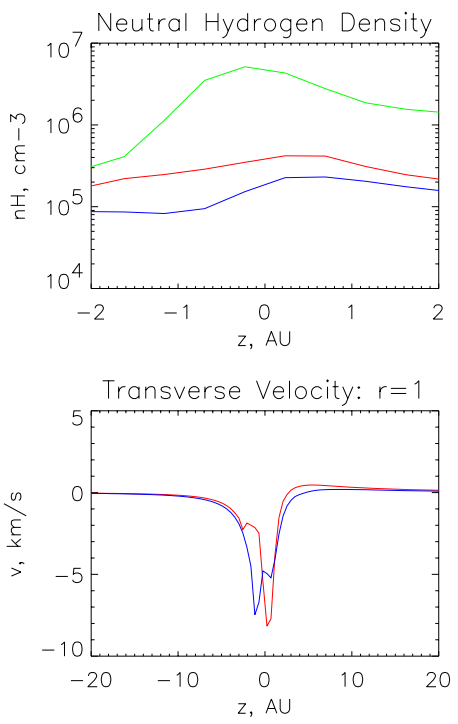

Fig. 4. Number densities, axial and radial components of velocity of the neutral gas-loaded flow versus axial distanse $z$, at a radial coordinate $r=1$ are shown by red curves. Here $n_{i}=$ $10^{5} \mathrm{~cm}^{-3}, V_{i}=26 \mathrm{~km} / \mathrm{s}, T_{i}=100 \mathrm{~K}$, $\beta_{0}=\beta_{\phi}=8 \cdot 10^{-8} \mathrm{~s}^{-1}, \omega=1$ at $1 \mathrm{AU}$ and $R_{\min }=1 \mathrm{AU}$, respectively. For comparison, results for the case without gas-loading also are shown by blue curves. At the top right panel different cases, without gas-loading, with gasloading at $\omega=1$ and gas-loading at $\omega=10$ are shown by blue, red and green curves, respectively. Everywhere $\mu=0.1$.
(1992) with a flattened slope $h=2.5$. It means that decreased values of the $Z_{E}$ by the factor of 100-1000 may cause different situations, when $R_{\max } \simeq 1 \mathrm{AU}$.

It should be noted here, that the above values of the $Z_{E}$ are calculated for pure species (Delsemme, 1982), while an influence of a solid phase matrix, where they are frozen-in, may be essential. For example, desorption of methane trapped in amorphous water ice is greatly suppressed by this effect; the critical sublimation temperature of $\simeq 50 \mathrm{~K}$ for pure $\mathrm{CH}_{4}$ increases to $\simeq 150 \mathrm{~K}$ for the case of $\mathrm{CH}_{4}$ frozen-in an $\mathrm{H}_{2} \mathrm{O}$ matrix (Hiraoka et al., 1998). In other words, thermal desorption of the "dirty" ice may be different with the result of a strongly reduced evaporation rate.

This case for methane may be important because its abundance in the icy mantles of dust grains in molecular clouds, unlike in comets, is more than $20 \%$ of water (Yeghikyan et al., 2001), while the evaporation rate (at the corresponding critical sublimation temperatures) is 6 times greater than for water (Delsemme, 1982). Further theoretical and laboratory work which investigates ice mixture sublimations at different temperature regime is needed to define more precisely their influence on the dust-gas flow dynamics near the Earth.

Thus, we conclude that gas-loading of the gas flow by the dust molecules evaporated from the dust grains may perhaps also increase the total amount of gaseous matter accreted by the Earth. For the density of incoming flow of about $10^{5} \mathrm{~cm}^{-3}$ and for conditions, shown in Fig. 4, they are about $3 \cdot 10^{21}, 6 \cdot 10^{21}$ and $7 \cdot 10^{22} \mathrm{~g}$, per 1 passage through the dense core of GMC for the cases without gas-loading, gas-loading at $\omega=1$ and gas-loading at $\omega=10$, respectively. Such amount of accreted neutral gas is quite enough to completely remove all $4.4 \cdot 10^{43}$ oxygen atoms (in the form of $\mathrm{O}_{2}$ ) of the present atmosphere, by the mechanism of Yabushita and Allen (1983) mentioned in Sect. 3. In a more recent paper Yabushita and Allen (1997) have pointed out that the reduced abundance of oxygen contained in ancient amber may be a record of such an event about $65 \mathrm{Myr}$ ago.

About $1 \%$ of dropped-out matter is dust, with probable refractory organic species in the mantle (roughly half the material). It would cause an annual accretion rate of dust of about $1 \cdot 10^{13} \mathrm{~g} / \mathrm{yr}$ (corresponding to the case without gasloading), which, by order of magnitude, is less than the amount necessary to rapidly cool the Earth (Yabushita and Allen, 1985, and references therein). On the other hand, in the case with gas-loading, even the small values of $\omega=1$ or 10 , by 2-3 orders of magnitude less than the maximum possible value which allows the gas-loading, $\omega=10^{3}$, would greatly increase neutral hydrogen fluxes at the Earth, up to $10^{13}$ atoms $/ \mathrm{sm}^{2} / \mathrm{s}$, which is greater by 7 orders of magnitude, than at present. This highly reactive hydrogen could reach stratospheric heights at $40-50 \mathrm{~km}$ by direct inflow and accumulate to levels that may be relevant for ozone depletion, and inevitably cause strong influence on the terrestrial climate (Fahr, 1968a, b; Bzowski et al., 1996).

\section{Conclusions}

In this paper we have shown that efficient dust evaporation processes increase the inflow density and push the heliopause down to the Earth, with the result that counterstreaming IC material directly influences the Earth. Investigation of the ionization structure of the inflowing material shows that cloud matter near the Earth is only weakly ionized. At IC $\mathrm{H}$-atom densities of the order of $n_{H}=10^{2}-10^{3} \mathrm{~cm}^{-3}$, incoming matter when approaching the Sun, but yet outside the reduced heliopause, is only subject to photoionization processes which simplify the hydrodynamic model of the flow. Thus, we have carried out the time-dependent simulation of neutral flow parameters by means of the 2-D gas dynamic numerical code, which takes into account both photoionization and gravity of the Sun. These processes are important to quantitatively estimate the total amount of neutral material, 
accreted from the cloud by the Earth during the pass-over time of about 1 million years. We have estimated this amount to be greater than $2 \cdot 10^{16} \mathrm{~g}$ per 1 passage, about $1 \%$ of which is in the form of dust $\left(\mathrm{H}_{2} \mathrm{O}\right.$ "dirty" ice or silicate grains, or both) with probable refractory organic species in the mantle (Yeghikyan et al., 2001). It may be important as a source of accumulated prebiotic material on the Earth during the manifold passages of the solar system through similar interstellar clouds.

We have also shown that large dust grains of the cloud, when present in countable abundance, serve as a source of neutrals, generated by the solar UV irradiation of dust particles, followed by a strong influence on the gas flow, by mass loading. It is shown that all kinds of dust grain populations with large icy mantles (grain radii are $0.5,3$ and $30 \mu \mathrm{m}$, respectively), discussed in the literature, are able to significantly gas-load the neutral gas flow with the result of a more than one order of magnitude increase in the gas density of neutrals near the Earth, as compared with the case without gas-loading.

Finally, it should be mentioned that work is in progress to incorporate into the model both the solar wind flow interactions and changes in chemistry in the inflowing gas and dust, bearing in mind probable contaminations of the Earth's atmosphere.

Acknowledgements. It is a pleasure for (A.G.Y) to acknowledge the receipt of financial support from the Alexander von Humboldt Foundation and to thank the Institute of Astrophysics and Extraterrestrial Research, University of Bonn for hospitality while this work was developed. The authors thank K. Scherer and I. Chashey for enlightening discussions, and R. J. LeVeque for making CLAWPACK available to the community. We are very grateful to two anonymous referees because of very helpful comments they made which improved the quality of this paper.

Topical Editor R. Forsyth thanks two referees for their help in evaluating this paper.

\section{References}

Allen's Astrophysical Quantities: (Ed) Cox, A. N., Springer, 2000.

Baranov, V. B., Krasnobaev, K. V, and Kulikovsky, A. G.: A model of the interaction of the solar wind with the interstellar medium, Sov. Phys. Dokl., 15, 791-793, 1971.

Baranov, V. B. and Malama, Yu. G.: Model of the solar wind interaction with the Local Interstellar Medium: numerical solution of self-consistent problem, J. Geophys. Res., 98, 15 157-15 163, 1993.

Bhatt, H. C.: Large dust grains in the dark cloud B5, Mon.Not.R.A.S, 222, 383-391, 1986.

Biermann, L., Brosowski, B. and Schmidt, U.: The interaction of the solar wind with a comet, Solar Physics, 1, 254-284, 1967.

Butler, D. M., Newman, M. J., and Talbot, Jr. R. J.: Interstellar cloud material: contribution to Planetary Atmospheres, Science, 201, 522-525, 1978.

Bzowski, M., Fahr, H.-J., and Rucinski, D.: Interplanetary neutral particle fluxes influencing the Earth's atmosphere and the terrestrial enviromnent, Icarus, 124, 209-219, 1996.
Clube, S. V. M. and Napier, W. M.: Giants comets and the Galaxy: implications of the terrestrial record, in: "The Galaxy and the Solar System", (Eds) Smoluchowski, R., Bahcall, J. N., and Mattews, M. S., The Univ. Arizona Press, Tucson, 69-82, 1986.

Delsemme, A. H.: Chemical composition of cometary nuclei, in: Comets, (Ed) Wilkening, L. L., Univ. Arizona Press, Tucson, Arizona, 85-130, 1982.

Fahr, H. J.: On the influence of neutral interstellar matter on the upper atmosphere, Astrophys. Space Sci., 2, 474-495, 1968a.

Fahr, H. J.: Neutral corpuscular energy flux by charge-transfer collisions in the vicinity of the Sun, Astrophys. Space Sci., 2, 496503, 1968 b.

Fahr, H. J.: Aspekte zur Wechselwirkung von Sternen mit dichten interstellaren Gaswolken, Mitt. Astron. Gesellschaft, 47, 233276, 1980.

Fahr, H.: Formation of the Heliospheric Boundaries and the Induced Dynamics of the Solar System: A Multifluid View, in: "The Outer Heliosphere: Beyond the Planets", (Eds) Scherer, K., Fichtner, H., and Marsch, E., Copernicus, Katlenburg-Lindau, 67-89, 2000.

Fahr, H. J.: Solar wind heating by an embedded quasi-isothermal pick-up ion fluid, Ann. Geophysicae, 20, 1509-1518, 2002.

Fahr, H. J. and Chashei, I.: On the thermodynamics of MHD waveheated solar wind protons, Astron. Astrophys., 395, 991-1000, 2002.

Fahr, H. J. and Rucinski, D.: Modification of properties and dynamics of distant solar wind due to its interaction with neutral interstellar gas, Nonlin. Proc. Geophys., 9, 377-384, 2002.

Fahr, H. J., Ripken, H. W., and Lay, G.: Plasma-dust interaction in the solar vicinity and their observational consequences, Astron. Astrophys., 102, 359-370, 1981.

Fahr, H. J., Grzedzielski, S. and Ratkiewicz, R.: Magnetohydrodynamic modeling of the 3-dimensional heliopause using the Newtonian approximation, Ann. Geophysicae, 6, 337-354, 1988.

Fahr, H. J., Kausch, T., and Scherer, H.: A 5-fluid hydrodynamic approach to model the solar system-interstellar medium interaction, Astron. Astrophys., 357, 268-282, 2000.

Farley, K. A. and Patterson, D. B.: A 100-kyr periodicity in the flux of extraterrestrial ${ }^{3} \mathrm{He}$ to the sea floor, Nature, 378, 600-602, 1995.

Frisch, P. C.: LISM structure - fragmented superbubble shell? Space Sci. Rev., 78, 213-222, 1996.

Greenberg, J. M.: Chemical evolution of interstellar dust - a source of prebiotic material?, in: Comets and the Origin of Life, (Ed) Ponnamperuma, C., 111-127, D. Reidel Publ. Comp., 1981.

Guinan, E. F. and Ribas, I.: Our changing Sun: the role of solar nuclear evolution and magnetic activity on Earth's atmospheres and climate, in: The evolving Sun and its influence on planetary environments, ASP Conf. Series, 269, 85-106, 2002.

Heiles, C., Goodman, A. A., McKee, C. F., and Zweibel, E. G.: Magnetic fields in dense regions, in: Fragmentatation of molecular clouds and star formation, (Eds) Falgarone, E., Boulander, F., and Duvert,G., IAU Symp. 147, Kluwer AP, 43-60, 1991.

Hiraoka, K., Miyagoshi, T., Takayama, T., Yamamoto, K. and Kihara, Y.: Gas-grain processes for the formation of $\mathrm{CH}_{4}$ and $\mathrm{H}_{2} \mathrm{O}$ : reactions of $\mathrm{H}$ atoms with $\mathrm{C}, \mathrm{O}$, and $\mathrm{CO}$ in the solid phase at 12 K, Astrophys. J., 498, 710-715, 1998.

Holzer, T. E.: Neutral hydrogen in interplanetary space, Rev. Geophys. Space Phys., 15, 467-490, 1977.

Hunt, R.: A fluid dynamical study of the accretion process, Mon. Not. R. Astron. Soc., 154, 141-165, 1971.

Kim, S.-H., Martin, P. G., and Hendry, P. D.: The size distribution 
of interstellar dust particles as determined from extinction, Astrophys. J., 422, 164-175, 1994.

Krasnobaev, K. V.: Static solar HII zone, Soviet Astronomy, 14, 840-844, 1971.

Lammer, H., Stumptner, W., Molina-Cuberos, G. J, Lara, L. M., and Tehrany, M. G.: From atmospheric isotope anomalies to a new perspective on early solar activity: consequences for planetary paleoatmospheres, in: The evolving Sun and its influence on planetary environments, ASP Conf. Series, 269, 85-106, 2002.

LeVeque, R. J.: Nonlinear conservation laws and finite volume methods, in: Computational methods for astrophysical fluid flow, (Eds) LeVeque R. J., Mihalas, D., Dorfi, E. A., and Muller, E., Saas-Fee Advanced Course 27, Springer, 1998.

McKee, C. F.: The multiphase interstellar medium, in: "The Physics of the Interstellar medium and Intergalactic Medium", (Eds) Ferrara, A., McKee, C. F., Heiles, C., and Shapiro, P. R., ASP Conf. Ser., 80, 292-316, 1995.

Maiolino, R. and Natta, A.: Large grains in our and external galaxies, Astrophys. Space Sci., 281, 233-242, 2002.

Maiolino, R., Marconi, A. and Oliva, E.: Dust in active nuclei II. Powder or gravel? Astron. Astrophys., 365, 37-48, 2001.

Mathis, J. S.: Dust models with tight abundance constraints, Astrophys. J, 472, 643-655, 1996.

Mathis, J. S., Rumpl, W., and Nordsieck, K. H.: The size distribution of interstellar grains, Astrophys. J., 217, 425-433, 1977.

Parker, E. N.: Interplanetary dynamical processes, Interscience Publishers, 1963.

Pauls H. L., Zank, G. P., and Williams, L. L.: Interaction of the solar wind with the local interstellar medium, J. Geophys. Res., 100, 21 595-21 604, 1995.

Ratkiewicz, R.: An analytical solution for the heliopause boundary and its comparison with numerical solutions, Astron. Astrophys., 255, 383-387, 1992.

Rawlings, J. M. C., Hartquist, T. W., Menten, K. M., and Williams, D. A.: Direct diagnosis of infall in collapsing protostars. -I. The theoretical identification of molecular species with broad velocity distributions, Mon. Not. R. Astron. S., 255, 471-485, 1992.

Ripken, H. W. and Fahr H. J.: Solar wind interactions with neutral hydrogen inside the orbit of the Earth, in: "Solar Wind Four Conf. Burghausen, Aug. 28 - Sept. 1, 1978 ”, 528-534, 1981.

Rowan-Robinson, M.: Interstellar dust in galaxies, Mon. Not. R. Astron. Soc., 258, 787-799, 1992.

Sackmann, I. and Boothroyd, A. I.: Our Sun. V. A bright young Sun consistent with helioseismology and warm temperatures on ancient Earth and Mars, Astrophys. J., 583, 1024-1039, 2003.

Scherer, K.: Variation of the heliospheric shield and its influence on Earth, in: "The Outer Heliosphere: Beyond the Planets", (Eds) Scherer, K., Fichtner, H., and Marsch, E., Copernicus, Katlenburg-Lindau, 327-356, 2000.

Scherer, K., Fichtner H., and Stawicki O.: Shielded by the wind: the influence of the interstellar medium on the environment of Earth, J. Atmosph. Solar-Terr. Phys., 64, 795-804, 2002.

Sciama, D. W.: On the interaction between cosmic rays and dark matter molecular clouds in the Milky Way - I. Basic considera- tions, Mon. Not. R. Astron. Soc., 312, 33-38, 2000.

Scoville, N. Z. and Sanders, D. B.: Observational constraints on the interaction of giant molecular clouds with the Solar System, in: "The Galaxy and the Solar System", (Eds) Smoluchowski, R., Bahcall, J. N., and Mattews, M. S., The Univ. Arizona Press, Tucson, 69-82, 1986.

Shima, E., Matsuda, T., Takeda, H. and Sawada, K.: Hydrodynamic calculations of axisymmetric accretion flow, Mon. Not. R. Astron. Soc., 217, 367-386, 1985.

Shima, E., Matsuda, T., and Inaguchi, T.: Interaction between a stellar wind and an accretion flow, Mon. Not. R. Astron. Soc., 221, 687-706, 1986.

Spitzer, Jr., L.: Physical Processes in the Interstellar Medium, A Wiley-Int. Publ., 1978.

Smith, R. G., Selgren, K., and Tokunaga, A. T.: A study of $\mathrm{H}_{2} \mathrm{O}$ ice in the micron spectrum of $\mathrm{OH} 2318+4.2(\mathrm{OH} 0739-14)$, Astrophys. J., 334, 209-219, 1988.

Talbot, Jr., R. J. and Newman, M. J.: Encounters between stars and dense interstellar clouds, Astroph. J. Suppl., 34, 295-308, 1977.

Whang, Y. C.: Ionization of interstellar hydrogen, Astrophys. J., 468, 947-954, 1996.

Wilkin, F. P.: Exact analytic solutions for stellar wind bow shocks, Astrophys. J., 459, L31-L34, 1996.

Williams, D. A.: Physical and chemical processes on dust, in: Dust and Chemistry in Astronomy, (Eds) Millar, T. J. and Williams, D. A., 143-170, Inst. Phys. Publ., 1993.

Williams, R. E.: The size of a solar HII region, Astrophys. J., 142, 314-320, 1965.

Wimmer-Schweingruber, R. F. and Bochsler, P.: Is there a record of interstellar pick-up ions in lunar soils? in: Acceleration and Transport of Energetic Particles Observed in the Heliosphere: ACE 2000 Symposium, (Eds) Mewaldt, R. A., et al., 270-273, 2000.

Yabushita, S. and Allen, A. J.: On the effect of interstellar gas on atmospheric oxygen and terrestrial life, Observatory, 103, 249251, 1983.

Yabushita, S. and Allen, A. J.: On the effect of interstellar matter on terrestrial climate, Observatory, 105, 198-200, 1985.

Yabushita, S. and Allen, A. J.: On the effect of accreted interstellar matter on the terrestrial environment, Mon. Not. R. Astron. Soc., 238, 1465-1478, 1989.

Yabushita, S. and Allen, A.J., Did an impact alone kill the dinosaurs?, Astronomy and Geophysics, 38, 15-19, 1997.

Yeghikyan, A. G., Viti, S., and Williams, D. A.: The formation of heavy hydrocarbons in molecular clouds, Mon. Not. R. Astron. Soc., 326, 313-316, 2001.

Zahnle, K. J. and Walker, J. C. G.: The evolution of solar ultraviolet luminosity, Rev. Geophys. Space Phys., 20, 280-292, 1982.

Zank, G. P.: Interaction of the solar wind with the local interstellar medium: a theoretical perspective, Space Sci. Rev., 89, 413-688, 1999.

Zank, G. P. and Frisch, P. C.: Consequences of a change in the galactic environment of the Sun, Astrophys. J., 518, 965-973, 1999. 